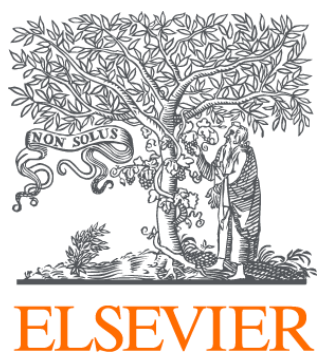

Since January 2020 Elsevier has created a COVID-19 resource centre with free information in English and Mandarin on the novel coronavirus COVID-

19. The COVID-19 resource centre is hosted on Elsevier Connect, the company's public news and information website.

Elsevier hereby grants permission to make all its COVID-19-related research that is available on the COVID-19 resource centre - including this research content - immediately available in PubMed Central and other publicly funded repositories, such as the WHO COVID database with rights for unrestricted research re-use and analyses in any form or by any means with acknowledgement of the original source. These permissions are granted for free by Elsevier for as long as the COVID-19 resource centre remains active. 


\title{
Intentions to participate in cervical and colorectal cancer screening during the COVID-19 pandemic: A mixed-methods study
}

\author{
Rebecca Wilson ${ }^{\mathrm{a}, *}$, Harriet Quinn-Scoggins ${ }^{\mathrm{b}}$, Yvonne Moriarty ${ }^{\mathrm{a}}$, Jacqueline Hughes ${ }^{\mathrm{a}}$, \\ Mark Goddard ${ }^{\mathrm{a}}$, Rebecca Cannings-John ${ }^{\mathrm{a}}$, Victoria Whitelock ${ }^{\mathrm{c}}$, Katriina L. Whitaker ${ }^{\mathrm{d}}$, \\ Detelina Grozeva $^{\text {a }}$, Julia Townson ${ }^{a}$, Kirstie Osborne ${ }^{c}$, Stephanie Smits ${ }^{\mathrm{e}}$, Michael Robling ${ }^{\mathrm{a}, \mathrm{f}}$, \\ Julie Hepburn $^{\mathrm{g}}$, Graham Moore ${ }^{\mathrm{f}}$, Ardiana Gjini ${ }^{\mathrm{h}, \mathrm{i}}$, Kate Brain ${ }^{\mathrm{b}, 1}$, Jo Waller ${ }^{\mathrm{j}, 1}$ \\ ${ }^{\text {a }}$ Centre for Trials Research, Cardiff University, Neuadd Meirionnydd, Heath Park, Cardiff CF14 4YS, UK \\ b PRIME Centre Wales, Division of Population Medicine, School of Medicine, Cardiff University, Neuadd Meirionnydd, Heath Park, Cardiff CF14 4YS, UK \\ ${ }^{\mathrm{c}}$ Cancer Intelligence, Cancer Research UK, 2 Redman Place, London, E20 1JQ, UK \\ ${ }^{\mathrm{d}}$ School of Health Sciences, University of Surrey, Guildford, UK \\ ${ }^{\mathrm{e}}$ Division of Population Medicine, School of Medicine, Cardiff University, Neuadd Meirionnydd, Heath Park, Cardiff CF14 4YS, UK \\ ${ }^{\mathrm{f}}$ DECIPHer (Centre for Development, Evaluation, Complexity and Implementation in Public Health Improvement), School of Social Sciences, Cardiff University, 1-3 \\ Museum Place, Cardiff, UK \\ ${ }^{g}$ Public Involvement Community, Health and Care Research Wales Support Centre, Castlebridge 4, 15-19, Cowbridge Road East, Cardiff CF11 $9 A B$, UK \\ ${ }^{\mathrm{h}}$ Public Health Wales, 2 Capital Quarter, Tyndall Street, Cardiff CF10 4BZ, UK \\ ${ }^{i}$ Cardiff University, Neuadd Meirionnydd, Heath Park, Cardiff CF14 4YS, UK \\ ${ }^{\mathrm{j}}$ Cancer Prevention Group, School of Cancer and Pharmaceutical Sciences, King's College London, Great Maze Pond, London SE1 9RT, UK
}

\section{A R T I C L E I N F O}

\section{Keywords:}

Cancer screening

Colorectal screening

Cervical screening

COVID-19

Mixed-methods study

\begin{abstract}
A B S T R A C T
Worldwide, cancer screening faced significant disruption in 2020 due to the COVID-19 pandemic. If this has led to changes in public attitudes towards screening and reduced intention to participate, there is a risk of long-term adverse impact on cancer outcomes. In this study, we examined previous participation and future intentions to take part in cervical and colorectal cancer (CRC) screening following the first national lockdown in the UK.

Overall, 7543 adults were recruited to a cross-sectional online survey in August-September 2020. Logistic regression analyses were used to identify correlates of strong screening intentions among 2319 participants eligible for cervical screening and 2502 eligible for home-based CRC screening. Qualitative interviews were conducted with a sub-sample of 30 participants. Verbatim transcripts were analysed thematically.

Of those eligible, $74 \%$ of survey participants intended to attend cervical screening and $84 \%$ intended to complete home-based CRC screening when next invited. Thirty percent and 19\% of the cervical and CRC samples respectively said they were less likely to attend a cancer screening appointment now than before the pandemic. Previous non-participation was the strongest predictor of low intentions for cervical (aOR 26.31, 95\% CI: 17.61-39.30) and CRC (aOR 67.68, 95\% CI: 33.91-135.06) screening. Interview participants expressed concerns about visiting healthcare settings but were keen to participate when screening programmes resumed.

Intentions to participate in future screening were high and strongly associated with previous engagement in both programmes. As screening services recover, it will be important to monitor participation and to ensure people feel safe to attend.
\end{abstract}

\footnotetext{
Abbreviations: CABS, Cancer Attitudes and Behaviour Study; CRC, colorectal cancer; aOR, Adjusted odds ratio; GP, General Practitioner.

* Corresponding author.

E-mail addresses: WilsonR13@Cardiff.ac.uk (R. Wilson), Quinn-ScogginsHD@Cardiff.ac.uk (H. Quinn-Scoggins), MoriartyY@Cardiff.ac.uk (Y. Moriarty),

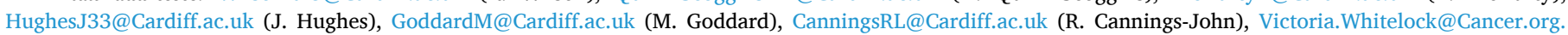

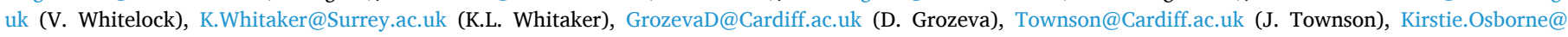

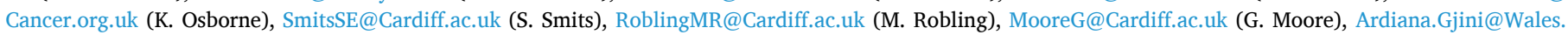
nhs.uk (A. Gjini), BrainKE@Cardiff.ac.uk (K. Brain), Jo.Waller@KCL.ac.uk (J. Waller).

1 Prof Kate Brain and Dr. Jo Waller share equally senior authorship of this manuscript.
} 


\section{Introduction}

Screening programmes for cervical and colorectal cancer (CRC) are part of the mainstay of cancer control in many countries (World Health Organization, 2017). The global Coronavirus pandemic saw unprecedented disruption to cancer screening in 2020, with national lockdowns and prioritisation of COVID-19 services causing many screening programmes to be paused.

In the UK, the three national cancer screening programmes were effectively paused between March and June 2020 when national lockdown restrictions were in place (Jones et al., 2020). It is estimated that around 3 million fewer people than normal in the UK had cancer screening between March and September 2020 (Cancer Intelligence Team, 2021). Disruption to cervical screening is predicted to cause over 600 excess cervical cancer cases in England (Castanon et al., 2021a) and changes across the care pathway for CRC led to an estimated 3500 fewer people beginning treatment between April and October 2020 (Morris et al., 2021). Modelling work in the Netherlands, Canada and Australia estimates an increase of 0.2 to $0.5 \%$ in CRC mortality if screening disruption continues and highlights the need for coverage to be rapidly restored to pre-2020 levels (de Jonge et al., 2021). It is essential that cancer screening continues to be seen as important and that public confidence in the safety of healthcare settings remains high, to avoid falling uptake and increased cancer burden.

Little is known about the impact of the pandemic on attitudes towards cancer screening or intentions to take part. Before the pandemic, screening intentions in the UK have been consistently high (and higher than actual uptake). In a 2016 population-based survey of screeningeligible British women, 88\% said they would attend when next invited (Marlow et al., 2017). In a study combining four population-based surveys of 60-70 year-olds in England carried out in 2014-16, 79-84\% of participants reported that they would definitely/probably complete CRC screening in the future (Vrinten et al., 2019). In one of these surveys, $64 \%$ of participants definitely intended to complete their next CRC screening kit (Dodd et al., 2019). Intentions were higher in a primary care based survey of people aged 45-59 in England who were not yet eligible for CRC screening: 74\% reported that they would definitely complete the kit (Smith et al., 2016).

Barriers vary between screening programmes, partly because cervical screening involves attending an appointment whereas CRC screening kits are posted for self-completion and involve stool sampling. Barriers to cervical and CRC screening and socio-demographic inequalities in uptake are well-established (Marlow et al., 2017; Douglas et al., 2016; Waller et al., 2009; Chapple et al., 2008; Palmer et al., 2014). The pandemic may have exacerbated existing practical barriers such as difficulty booking an appointment, and raised new emotional barriers reflecting concerns about COVID-19 infection risk and burdening the health service. In addition, it may have widened existing social inequalities, making screening harder for those disproportionately affected by the pandemic.

We present analyses from the population-based COVID-19 Cancer Attitudes and Behaviour Study (CABS) (Quinn-Scoggins et al., 2021) measuring intentions to take part in cervical and CRC screening following the first UK national lockdown. Understanding cancer screening barriers and intentions during the pandemic is an essential first step towards mitigating potential long-term adverse effects on screening participation.

\section{Methods}

We carried out a mixed-methods study including a cross-sectional population-based online survey and qualitative interviews with a subsample of survey participants. The methods are described in detail elsewhere (Quinn-Scoggins et al., 2021; CABS, 2021). The survey was carried out in August-September 2020 and the interviews in September-November 2020.

\subsection{Participants}

The online survey participants were English-speaking adults (aged 18+) living in the UK and recruited via Cancer Research UK's online panel provider, the HealthWise Wales database and social media. For this analysis, we used data from two sub-samples of survey respondents: people with a cervix aged 25-64 years (eligible for cervical screening) and people aged 60-74 years in England, Wales and Northern Ireland and age 50-74 in Scotland (eligible for CRC screening). Information on breast screening was only collected from participants recruited via HealthWise Wales, therefore analyses of these data are not presented here.

\subsection{Quantitative methods and analysis}

Outcome measures were intention to take part in cervical and CRC screening when next invited (see (CAM, 2021) for exact wording). Binary variables indicating strong intention to take part were created (Yes, definitely vs. Yes, probably/No, probably not/No, definitely not/Don't know). Responding 'Yes, definitely' has been shown to have a strong association with actual uptake (Power et al., 2008).

Potential explanatory variables were sex (for CRC screening), age, ethnicity, marital status, educational attainment, smoking status, UK country of residence, disability status and personal or family/friends' history of cancer. Participants were asked if they had experienced any of 13 barriers to cervical screening and 10 barriers to CRC screening (yes/ no) derived from the Cancer Research UK Cancer Awareness Measure 2019 (Hudson and Osborne, 2021) with COVID-specific items developed for this study. The number of barriers endorsed was summed to create a total barriers score for cervical (range: 0-13) and CRC (range: 0-10) screening. Attitudes to attending healthcare settings and concern about delays to cancer screening and diagnosis in the context of the COVID-19 pandemic were measured using six items (Ide-Walters and Moffat, 2021) (see Table 1). Participants who had not attended their last cervical screening were asked if this was due to coronavirus, with response options "No, not going was not related to coronavirus", "Yes, I tried to go but wasn't able to go due to coronavirus", "Yes, I chose not to go due to coronavirus".

A pre-specified analysis plan was published on Open Science Framework (CABS, 2021). Descriptive statistics were used to characterise the study samples. Explanatory variables were regressed onto each binary intention outcome. Bonferroni correction was used to adjust for multiple testing and a $99.9 \%$ confidence interval (CI) was used for bivariate regression models (alpha 0.001). All explanatory variables of interest were included in the multivariable models. Sample weights were included in the bivariate and multivariable models. Analysis was performed using Stata 16 (StataCorp, 2019).

\subsection{Qualitative methods and analysis}

Semi-structured telephone interviews were conducted with survey participants who had consented to be recontacted. We used purposive sampling to ensure a range of participants with respect to age, gender and symptom experience (the primary outcome in the main study). Interview participants received a $£ 20$ high-street voucher.

A topic guide was used to explore participants' experiences of cancer symptoms and help-seeking, screening and health-related behaviours in the previous six months (Supplementary Material 1). Where participants had not been invited to or taken part in cancer screening during this period, they were asked to consider hypothetically how they might have responded if they had been invited. Interviews were transcribed verbatim and anonymised. Data were analysed thematically (Braun and Clarke, 2006) using NVivo12 (QSR International Pty Ltd, 2018) with $20 \%$ double coded. The analysis presented here relates to screeningrelated themes only. 
Table 1

Sample characteristics for cervical screening-eligible and CRC screening-eligible participants in the UK, August-September 2020.

\begin{tabular}{|c|c|c|c|}
\hline & & $\begin{array}{l}\text { Cervical } \\
\text { screening } \\
\text { eligible } \\
\text { sample ( } N= \\
2319)\end{array}$ & $\begin{array}{l}\text { CRC } \\
\text { screening } \\
\text { eligible } \\
\text { sample }(N= \\
2502)\end{array}$ \\
\hline \multirow[t]{3}{*}{ Gender } & Male & 0 & $1572(62.8 \%)$ \\
\hline & Female & $2306(99.4 \%)$ & 927 (37.1\%) \\
\hline & Other & $13(0.6 \%)$ & $3(0.1 \%)$ \\
\hline \multirow[t]{5}{*}{ Age } & $25-34$ & $607(26.2 \%)$ & 0 \\
\hline & $35-44$ & $649(28.0 \%)$ & 0 \\
\hline & $45-54$ & $596(25.7 \%)$ & $33(1.3 \%)^{1}$ \\
\hline & $55-64$ & $467(20.1 \%)$ & $718(28.7 \%)$ \\
\hline & $65-74$ & 0 & $1751(70.0 \%)$ \\
\hline \multirow[t]{3}{*}{ Ethnicity } & White & $2005(86.5 \%)$ & $2419(96.7 \%)$ \\
\hline & Ethnic minorities ${ }^{2}$ & $312(13.5 \%)$ & $79(3.2 \%)$ \\
\hline & Missing & $2(0.01 \%)$ & $4(0.2 \%)$ \\
\hline \multirow[t]{4}{*}{ Relationship status } & $\begin{array}{l}\text { Married/in a } \\
\text { relationship }\end{array}$ & $1603(69.1 \%)$ & $1745(69.7 \%)$ \\
\hline & $\begin{array}{l}\text { Single/ never } \\
\text { married }\end{array}$ & $478(20.6 \%)$ & $234(9.4 \%)$ \\
\hline & $\begin{array}{l}\text { Divorced/ } \\
\text { separated/ widowed }\end{array}$ & $228(9.8 \%)$ & $515(20.6 \%)$ \\
\hline & Missing & $10(0.4 \%)$ & $8(0.3 \%)$ \\
\hline \multirow[t]{4}{*}{ Educational attainment } & $\begin{array}{l}\text { Degree/ higher } \\
\text { degree }\end{array}$ & $942(40.6 \%)$ & $907(36.3 \%)$ \\
\hline & $\begin{array}{l}\text { Higher education } \\
\text { qualification/ A- } \\
\text { levels or Highers/ } \\
\text { ONC/ BTEC }\end{array}$ & $782(33.7 \%)$ & $773(30.9 \%)$ \\
\hline & $\begin{array}{l}\text { O level/ GCSEs/ } \\
\text { No formal } \\
\text { qualifications }\end{array}$ & $552(23.8 \%)$ & 767 (30.7\%) \\
\hline & Missing & $43(1.9 \%)$ & $55(2.2 \%)$ \\
\hline \multirow[t]{4}{*}{ Smoking status } & Never smoked & $1159(50.0 \%)$ & $1144(45.7 \%)$ \\
\hline & Used to smoke & $611(26.4 \%)$ & $1081(43.2 \%)$ \\
\hline & $\begin{array}{l}\text { Smoke every day/ } \\
\text { not every day }\end{array}$ & $528(22.8 \%)$ & $246(9.8 \%)$ \\
\hline & Missing & $21(0.9 \%)$ & $31(1.2 \%)$ \\
\hline \multirow[t]{5}{*}{ UK nation } & Wales & $455(19.6 \%)$ & $1030(41.2 \%)$ \\
\hline & Scotland & $140(6.0 \%)$ & $218(8.7 \%)$ \\
\hline & Northern Ireland & $38(1.6 \%)$ & $27(1.1 \%)$ \\
\hline & England & $1680(72.5 \%)$ & $1217(48.6 \%)$ \\
\hline & Missing & $6(0.3 \%)$ & $10(0.4 \%)$ \\
\hline \multirow[t]{3}{*}{ Disability } & No & $1892(81.6 \%)$ & $1985(79.3 \%)$ \\
\hline & Yes & 374 (16.1\%) & 475 (19.0\%) \\
\hline & Missing & $53(2.3 \%)$ & $42(1.7 \%)$ \\
\hline \multirow[t]{3}{*}{ Cancer history } & No & $522(22.5 \%)$ & 347 (13.9\%) \\
\hline & Yes, self & $117(5.1 \%)$ & 347 (13.9\%) \\
\hline & Yes, others ${ }^{3}$ & $1680(72.5 \%)$ & $1808(72.3 \%)$ \\
\hline \multirow{3}{*}{$\begin{array}{l}\text { Previous cervical/CRC } \\
\text { screening } \\
\text { participation }\end{array}$} & Yes & $1696(73.1 \%)$ & $2035(81.3 \%)$ \\
\hline & No & $516(22.3 \%)$ & $358(14.3 \%)$ \\
\hline & Missing & $107(4.6 \%)$ & $109(4.4 \%)$ \\
\hline \multirow{4}{*}{$\begin{array}{l}\text { Reason for non- } \\
\text { participation in } \\
\text { previous cervical } \\
\text { screening }(N=516)\end{array}$} & $\begin{array}{l}\text { No, not going was not } \\
\text { related to coronavirus }\end{array}$ & $363(70.4 \%)$ & \\
\hline & $\begin{array}{l}\text { Yes, I tried to go but } \\
\text { wasn't able to due to } \\
\text { coronavirus }\end{array}$ & $63(12.2 \%)$ & \\
\hline & $\begin{array}{l}\text { Yes, I chose not to go } \\
\text { due to coronavirus }\end{array}$ & $76(14.7 \%)$ & \\
\hline & Missing & $14(2.7 \%)$ & \\
\hline \multirow{5}{*}{$\begin{array}{l}\text { Screening intention } \\
\text { when next invited }\end{array}$} & Yes, definitely & $1704(73.5 \%)$ & $2109(84.3 \%)$ \\
\hline & Yes, probably & $283(12.2 \%)$ & $173(6.9 \%)$ \\
\hline & No, probably not & $128(5.5 \%)$ & 109 (4.4\%) \\
\hline & No, definitely not & $91(3.9 \%)$ & $52(2.1 \%)$ \\
\hline & Don't know & $113(4.9 \%)$ & $59(2.4 \%)$ \\
\hline \multirow{3}{*}{$\begin{array}{l}\text { I am confident that I } \\
\text { would be safe from } \\
\text { coronavirus if I } \\
\text { needed to attend an } \\
\text { appointment at a } \\
\text { hospital }\end{array}$} & $\begin{array}{l}\text { Strongly/ somewhat } \\
\text { agree }\end{array}$ & $1448(62.4 \%)$ & $1643(65.7 \%)$ \\
\hline & $\begin{array}{l}\text { Strongly/ somewhat } \\
\text { disagree }\end{array}$ & $563(24.3 \%)$ & $606(24.2 \%)$ \\
\hline & Missing & $308(13.3 \%)$ & $253(10.1 \%)$ \\
\hline \multirow{2}{*}{$\begin{array}{l}\text { I am confident that I } \\
\text { would be safe from } \\
\text { coronavirus if I }\end{array}$} & $\begin{array}{l}\text { Strongly/ somewhat } \\
\text { agree }\end{array}$ & $1569(67.7 \%)$ & $1888(75.5 \%)$ \\
\hline & & $461(19.9 \%)$ & $420(16.8 \%)$ \\
\hline
\end{tabular}

Table 1 (continued)

\begin{tabular}{|c|c|c|c|}
\hline & & $\begin{array}{l}\text { Cervical } \\
\text { screening } \\
\text { eligible } \\
\text { sample }(N= \\
2319)\end{array}$ & $\begin{array}{l}\text { CRC } \\
\text { screening } \\
\text { eligible } \\
\text { sample }(N= \\
2502)\end{array}$ \\
\hline $\begin{array}{l}\text { needed to attend an } \\
\text { appointment at my }\end{array}$ & $\begin{array}{l}\text { Strongly/ somewhat } \\
\text { disagree }\end{array}$ & & \\
\hline GP surgery & Missing & $289(12.5 \%)$ & $194(7.8 \%)$ \\
\hline $\begin{array}{l}\text { I am worried about } \\
\text { delays to cancer tests }\end{array}$ & $\begin{array}{l}\text { Strongly/ somewhat } \\
\text { agree }\end{array}$ & $1735(74.8 \%)$ & $1911(76.4 \%)$ \\
\hline $\begin{array}{l}\text { and investigations } \\
\text { caused by }\end{array}$ & $\begin{array}{l}\text { Strongly/ somewhat } \\
\text { disagree }\end{array}$ & $344(14.8 \%)$ & $399(16.0 \%)$ \\
\hline coronavirus & Missing & $240(10.4 \%)$ & $192(7.7 \%)$ \\
\hline $\begin{array}{l}\text { I am less likely to attend } \\
\text { a cancer screening }\end{array}$ & $\begin{array}{l}\text { Strongly/ somewhat } \\
\text { agree }\end{array}$ & $684(29.5 \%)$ & $466(18.6 \%)$ \\
\hline $\begin{array}{l}\text { appointment now } \\
\text { than I was before the }\end{array}$ & $\begin{array}{l}\text { Strongly/ somewhat } \\
\text { disagree }\end{array}$ & $1388(59.9 \%)$ & $1851(74.0 \%)$ \\
\hline $\begin{array}{l}\text { coronavirus } \\
\text { pandemic lockdown }\end{array}$ & Missing & $247(10.7 \%)$ & $185(7.4 \%)$ \\
\hline $\begin{array}{l}\text { I am worried about } \\
\text { delays to cancer }\end{array}$ & $\begin{array}{l}\text { Strongly/ somewhat } \\
\text { agree }\end{array}$ & $1730(74.6 \%)$ & $1880(75.1 \%)$ \\
\hline \multirow[t]{2}{*}{$\begin{array}{l}\text { screening caused by } \\
\text { coronavirus }\end{array}$} & $\begin{array}{l}\text { Strongly/ somewhat } \\
\text { disagree }\end{array}$ & $355(15.3 \%)$ & $427(17.1 \%)$ \\
\hline & Missing & $234(10.1 \%)$ & $195(7.8 \%)$ \\
\hline \multirow[t]{3}{*}{$\begin{array}{l}\text { Cancer screening saves } \\
\text { lives }\end{array}$} & $\begin{array}{l}\text { Strongly/ somewhat } \\
\text { agree }\end{array}$ & $2133(92.0 \%)$ & $2421(96.8 \%)$ \\
\hline & $\begin{array}{l}\text { Strongly/ somewhat } \\
\text { disagree }\end{array}$ & $84(3.6 \%)$ & $38(1.5 \%)$ \\
\hline & Missing & $102(4.4 \%)$ & $43(1.7 \%)$ \\
\hline
\end{tabular}

1 Only participants age 50-59 residing in Scotland included.

2 Includes: 'Mixed/multiple ethnic groups', 'Asian/Asian British', 'black/African/Caribbean/black British', 'other ethnic group'.

${ }^{3}$ Includes: Partner, someone in immediate family, someone in wider family, close friend, someone else in family or friends.

\section{Results}

\subsection{Quantitative results}

\subsubsection{Sample characteristics}

From the overall survey sample of 7543 , we included 2319 participants who were eligible for cervical screening and 2502 eligible for CRC screening. Participants who preferred not to state their intentions were excluded ( $n=36$ and $n=4$ for cervical and CRC screening respectively), as were those who reported that they would not be eligible for a future invitation (cervical: $n=159$; CRC: $n=45$ ). Participants eligible for both screening programmes $(n=1003)$ were included in both samples. Demographic characteristics of each sample are presented in Table 1. Selfreported participation in screening when last invited was high for both cervical (73\%) and CRC (81\%) screening. Of those who had not attended their last cervical screen, $70 \%(363 / 516)$ said this was unrelated to COVID-19. Twelve percent (63/516) reported being unable to attend despite trying and $15 \%(76 / 516)$ had chosen not to attend due to COVID-19.

\subsubsection{COVID-related attitudes (Table 1)}

As shown in Table 1, when asked how safe from coronavirus they would feel if attending an appointment at a hospital or GP surgery, over $60 \%$ of participants in both samples reported being confident they would be safe in either healthcare setting. Almost $75 \%$ in both samples reported that they were worried about delays to cancer tests and investigations, and to screening, caused by coronavirus. Thirty percent of the cervical sample and $19 \%$ of the CRC sample agreed that they were less likely to attend a cancer screening appointment now than before the pandemic. Over $90 \%$ of both samples agreed that cancer screening saves lives.

\subsubsection{Cervical and CRC screening intentions}

Most eligible respondents said they would 'definitely' participate in 
cervical (74\%) and CRC (84\%) screening when next invited (see Table 1 for a full breakdown of responses). Intention was strongly related to previous uptake: $91 \%$ of previous cervical screening attenders definitely intended to go when next invited compared with $24 \%$ of previous nonattenders. The figures were $97 \%$ and $20 \%$ for previous CRC completers and non-completers respectively. In cervical screening, intention also varied according to whether previous non-attendance was related to COVID-19. Only $14 \%(50 / 363)$ of women who had not attended for reasons unrelated to COVID-19 were intending to go when next invited. This figure was $36 \%(27 / 76)$ for those who had decided not to attend for COVID-related reasons and 70\% (44/63) for those who had been unable to attend due to COVID despite having tried.

\subsubsection{Barriers to cervical and CRC screening}

Participants reported between 0 and 13 barriers to cervical screening (mean $=0.68$; median $=0$ ) and between 0 and 7 barriers to CRC

Table 2

Unadjusted and adjusted logistic regression models predicting low future cervical screening intention, UK, August-September 2020 ( $n=2319$ ).

\begin{tabular}{|c|c|c|c|c|c|c|}
\hline & & \multirow{2}{*}{$\begin{array}{l}\mathrm{N}(\%) \text { reported Yes, probably/ No, } \\
\text { definitely/ probably/ Don't know }{ }^{1} \\
N=615\end{array}$} & \multicolumn{2}{|l|}{ Unadjusted models } & \multicolumn{2}{|l|}{$\begin{array}{l}\text { Adjusted model } \\
N=1561\end{array}$} \\
\hline & & & OR $(99.9 \% \mathrm{CI})$ & $\mathrm{p}^{*}$ & OR $(95 \% \mathrm{CI})$ & $\mathrm{p}^{* *}$ \\
\hline \multirow[t]{3}{*}{ Age } & $25-34$ & $192(31.6 \%)$ & 1.00 & & 1.00 & \\
\hline & $35-49$ & $243(26.1 \%)$ & $0.82(0.55-1.24)$ & 0.119 & $1.23(0.75-2.00)$ & 0.414 \\
\hline & $50-64$ & $180(23.1 \%)$ & $0.73(0.47-1.13)$ & 0.019 & $1.42(0.82-2.47)$ & 0.212 \\
\hline \multirow[t]{2}{*}{ Ethnicity } & White & $498(24.8 \%)$ & 1.00 & & 1.00 & \\
\hline & Ethnic minorities & $116(37.2 \%)$ & $1.74(1.10-2.76)$ & $<0.001$ & $1.08(0.58-2.00)$ & 0.814 \\
\hline \multirow[t]{3}{*}{ Marital status } & $\begin{array}{l}\text { Married/in a } \\
\text { relationship }\end{array}$ & $336(21.0 \%)$ & 1.00 & & 1.00 & \\
\hline & $\begin{array}{l}\text { Single/never } \\
\text { married }\end{array}$ & 207 (43.3\%) & $2.73(1.83-4.07)$ & $<0.001$ & $1.96(1.26-3.06)$ & 0.003 \\
\hline & $\begin{array}{l}\text { Divorced/ } \\
\text { separated/ } \\
\text { widowed }\end{array}$ & $68(29.8 \%)$ & $1.58(0.88-2.84)$ & 0.010 & $1.72(1.00-2.96)$ & 0.049 \\
\hline \multirow[t]{3}{*}{ Education } & $\begin{array}{l}\text { Degree or higher } \\
\text { degree }\end{array}$ & $245(26.0 \%)$ & 1.00 & & 1.00 & \\
\hline & $\begin{array}{l}\text { Mid-level } \\
\text { qualifications }\end{array}$ & $206(26.3 \%)$ & $0.99(0.66-1.47)$ & 0.909 & $1.08(0.70-1.65)$ & 0.738 \\
\hline & $\begin{array}{l}\text { Low/no } \\
\text { qualifications }\end{array}$ & $152(27.5 \%)$ & $1.08(0.70-1.68)$ & 0.541 & $0.70(0.42-1.16)$ & 0.170 \\
\hline \multirow[t]{3}{*}{ Smoking status } & Never smoked & $301(26.0 \%)$ & 1.00 & & 1.00 & \\
\hline & Ex-smoker & $131(21.4 \%)$ & $0.87(0.56-1.34)$ & 0.275 & $1.07(0.70-1.70)$ & 0.782 \\
\hline & Current smoker & $174(33.0 \%)$ & $1.47(0.97-2.32)$ & 0.002 & $1.14(0.71-1.84)$ & 0.579 \\
\hline \multirow[t]{4}{*}{ Region } & England & $478(28.5 \%)$ & 1.00 & & 1.00 & \\
\hline & Wales & $86(18.9 \%)$ & $0.61(0.40-0.95)$ & $<0.001$ & $0.53(0.32-0.85)$ & $<0.001$ \\
\hline & Scotland & $39(27.9 \%)$ & $0.99(0.52-1.91)$ & 0.975 & $0.82(0.39-1.72)$ & 0.593 \\
\hline & Northern Ireland & $12(31.6 \%)$ & $1.17(0.36-3.85)$ & 0.659 & $1.48(0.52-4.20)$ & 0.462 \\
\hline \multirow[t]{2}{*}{ Do you consider yourself to have a disability? } & No & $467(24.7 \%)$ & 1.00 & & 1.00 & \\
\hline & Yes & $128(34.2 \%)$ & $1.56(1.00-2.45)$ & 0.001 & $1.45(0.88-2.41)$ & 0.146 \\
\hline \multirow{3}{*}{$\begin{array}{l}\text { Have you, anyone in your family or any of your } \\
\text { friends had cancer? }\end{array}$} & No & $174(33.3 \%)$ & 1.00 & & 1.00 & \\
\hline & Yes, self & $30(25.6 \%)$ & $0.73(0.30-1.75)$ & 0.231 & $1.56(0.67-3.64)$ & 0.307 \\
\hline & Yes, other & $411(24.5 \%)$ & $0.72(0.49-1.07)$ & 0.007 & $1.23(0.77-1.98)$ & 0.385 \\
\hline \multirow{2}{*}{$\begin{array}{l}\text { Did you go for cervical screening the last time you } \\
\text { were invited? }\end{array}$} & Yes & $152(9.0 \%)$ & 1.00 & & 1.00 & \\
\hline & No & $394(76.4 \%)$ & $\begin{array}{l}34.34 \\
(20.98-56.22)\end{array}$ & $<0.001$ & $\begin{array}{l}26.37 \\
(17.66-39.37)\end{array}$ & $<0.001$ \\
\hline Total number barriers, mean (SD) & & $1.31(1.31)$ & $1.85(1.51-2.27)$ & $<0.001$ & $1.61(1.26-2.04)$ & $<0.001$ \\
\hline \multirow{2}{*}{$\begin{array}{l}\text { I am confident that I would be safe from } \\
\text { coronavirus if I needed to attend an } \\
\text { appointment at a hospital }\end{array}$} & $\begin{array}{l}\text { Strongly/somewhat } \\
\text { agree }\end{array}$ & $320(22.1 \%)$ & 1.00 & & 1.00 & \\
\hline & $\begin{array}{l}\text { Strongly/ somewhat } \\
\text { disagree }\end{array}$ & $190(33.8 \%)$ & $1.91(1.28-2.85)$ & $<0.001$ & $1.13(0.63-2.03)$ & 0.673 \\
\hline \multirow{2}{*}{$\begin{array}{l}\text { I am confident I would be safe from coronavirus if I } \\
\text { needed to attend an appointment at my GP } \\
\text { surgery }\end{array}$} & $\begin{array}{l}\text { Strongly/ somewhat } \\
\text { agree }\end{array}$ & $350(22.3 \%)$ & 1.00 & & 1.00 & \\
\hline & $\begin{array}{l}\text { Strongly/ somewhat } \\
\text { disagree }\end{array}$ & $168(36.4 \%)$ & $2.03(1.34-3.08)$ & $<0.001$ & $1.14(0.63-2.07)$ & 0.659 \\
\hline \multirow[t]{2}{*}{$\begin{array}{l}\text { I am worried about delays to cancer tests and } \\
\text { investigations caused by coronavirus }\end{array}$} & $\begin{array}{l}\text { Strongly/ somewhat } \\
\text { agree }\end{array}$ & $389(22.4 \%)$ & 1.00 & & 1.00 & \\
\hline & $\begin{array}{l}\text { Strongly/ somewhat } \\
\text { disagree }\end{array}$ & $141(41.0 \%)$ & $2.49(1.58-3.90)$ & $<0.001$ & $1.22(0.56-2.64)$ & 0.612 \\
\hline \multirow{2}{*}{$\begin{array}{l}\text { I am less likely to attend a cancer screening } \\
\text { appointment now than I was before the } \\
\text { pandemic }\end{array}$} & $\begin{array}{l}\text { Strongly/ somewhat } \\
\text { agree }\end{array}$ & $262(38.3 \%)$ & 1.00 & & 1.00 & \\
\hline & $\begin{array}{l}\text { Strongly/ somewhat } \\
\text { disagree }\end{array}$ & $256(18.4 \%)$ & $0.35(0.24-0.52)$ & $<0.001$ & $0.48(0.32-0.71)$ & $<0.001$ \\
\hline \multirow[t]{2}{*}{$\begin{array}{l}\text { I am worried about delays to cancer screening } \\
\text { caused by coronavirus }\end{array}$} & $\begin{array}{l}\text { Strongly/somewhat } \\
\text { agree }\end{array}$ & $383(22.1 \%)$ & 1.00 & & 1.00 & \\
\hline & $\begin{array}{l}\text { Strongly/ somewhat } \\
\text { disagree }\end{array}$ & $143(40.3 \%)$ & $2.52(1.60-3.97)$ & $<0.001$ & $2.24(1.02-4.94)$ & 0.046 \\
\hline \multirow[t]{2}{*}{ Cancer screening saves lives } & $\begin{array}{l}\text { Strongly/ somewhat } \\
\text { agree }\end{array}$ & $514(24.1 \%)$ & 1.00 & & 1.00 & \\
\hline & $\begin{array}{l}\text { Strongly/ somewhat } \\
\text { disagree }\end{array}$ & $46(54.8 \%)$ & $3.20(1.47-6.97)$ & $<0.001$ & $0.92(0.37-2.26)$ & 0.852 \\
\hline
\end{tabular}

\footnotetext{
${ }^{1}$ Compared with 'yes, definitely'.

* $p<0.001$.

$p<0.01$.
} 
screening (mean $=0.23$; median $=0$ ) (see Supplementary Material 2). The most frequently reported barriers to cervical screening were worry about pain (12\%), a previous bad experience (9\%) and embarrassment (9\%), although for previous non-attenders embarrassment was the most reported barrier (23\%). Worry about catching COVID-19 was not frequently endorsed ( $4 \%$ overall; $10 \%$ in previous non-attenders). The most common barriers to CRC screening were finding it too messy (5\%), not having symptoms (4\%) and embarrassment (4\%). Barriers were

Table 3

Unadjusted and adjusted logistic regression models predicting low future CRC screening intention, UK, August-September 2020 ( $n=2502$ ).

\begin{tabular}{|c|c|c|c|c|c|c|}
\hline & & \multirow{2}{*}{$\begin{array}{l}\text { N (\%) reported yes, probably/ no, } \\
\text { definitely/ probably/ Don't know } N \\
=393\end{array}$} & \multicolumn{2}{|l|}{ Unadjusted models } & \multicolumn{2}{|c|}{$\begin{array}{l}\text { Multiply adjusted model } \\
N=1768\end{array}$} \\
\hline & & & OR $(99.9 \% \mathrm{CI})$ & $\mathrm{p}^{*}$ & OR $(95 \% \mathrm{CI})$ & $\mathrm{p}^{* *}$ \\
\hline \multirow[t]{2}{*}{ Gender } & Male & $248(15.8 \%)$ & 1.00 & & 1.00 & 0.736 \\
\hline & Female/other & $145(15.6 \%)$ & $0.89(0.56-1.39)$ & 0.381 & $1.10(0.62-1.98)$ & \\
\hline \multirow[t]{3}{*}{ Age } & $50-54$ & $9(27.3 \%)$ & 1.00 & & 1.00 & \\
\hline & $55-64$ & $148(20.6 \%)$ & $0.79(0.21-3.03)$ & 0.571 & $0.59(0.07-4.82)$ & 0.619 \\
\hline & $65-74$ & $236(13.5 \%)$ & $0.51(0.14-1.90)$ & 0.092 & $0.50(0.06-3.98)$ & 0.515 \\
\hline \multirow[t]{2}{*}{ Ethnicity } & White & $381(15.8 \%)$ & 1.00 & & 1.00 & \\
\hline & Ethnic minorities & $11(13.9 \%)$ & $1.10(0.31-3.87)$ & 0.809 & $0.16(0.03-0.94)$ & 0.043 \\
\hline \multirow[t]{3}{*}{ Marital status } & $\begin{array}{l}\text { Married/ in a } \\
\text { relationship }\end{array}$ & $231(13.2 \%)$ & 1.00 & & 1.00 & \\
\hline & $\begin{array}{l}\text { Single/never } \\
\text { married }\end{array}$ & $60(25.6 \%)$ & $\begin{array}{l}2.44 \\
(1.28-4.63)\end{array}$ & $<0.001$ & $1.22(0.52-2.84)$ & 0.653 \\
\hline & $\begin{array}{l}\text { Divorced/ } \\
\text { separated/ } \\
\text { widowed }\end{array}$ & $101(19.6 \%)$ & $1.58(0.93-2.70)$ & 0.004 & $0.90(0.46-1.78)$ & 0.762 \\
\hline \multirow[t]{3}{*}{ Education } & $\begin{array}{l}\text { Degree or higher } \\
\text { degree }\end{array}$ & $120(13.2 \%)$ & 1.00 & & 1.00 & \\
\hline & $\begin{array}{l}\text { Mid-level } \\
\text { qualifications }\end{array}$ & $127(16.4 \%)$ & $1.18(0.67-2.07)$ & 0.343 & $1.16(0.58-2.33)$ & 0.681 \\
\hline & $\begin{array}{l}\text { Low/no } \\
\text { qualifications }\end{array}$ & $139(18.1 \%)$ & $1.47(0.85-2.54)$ & 0.021 & $1.36(0.73-2.54)$ & 0.339 \\
\hline \multirow[t]{3}{*}{ Smoking status } & Never smoked & $175(15.3 \%)$ & 1.00 & & 1.00 & \\
\hline & Ex-smoker & $162(15.0 \%)$ & $1.01(0.63-1.63)$ & 0.918 & $0.94(0.50-1.76)$ & 0.839 \\
\hline & Current smoker & $55(22.4 \%)$ & $1.33(0.67-2.65)$ & 0.173 & $0.99(0.38-2.58)$ & 0.980 \\
\hline \multirow[t]{4}{*}{ Region } & England & $223(18.3 \%)$ & 1.00 & & 1.00 & \\
\hline & Wales & $115(11.2 \%)$ & $\begin{array}{l}0.59 \\
(0.38-0.91)\end{array}$ & $<0.001$ & $0.86(0.52-1.43)$ & 0.563 \\
\hline & Scotland & $43(19.7 \%)$ & $1.22(0.65-2.29)$ & 0.308 & $1.30(0.50-3.40)$ & 0.587 \\
\hline & Northern Ireland & $9(33.3 \%)$ & $2.26(0.54-9.42)$ & 0.060 & $1.54(0.11-20.75)$ & 0.747 \\
\hline \multirow[t]{2}{*}{ Do you consider yourself to have a disability? } & No & $282(14.2 \%)$ & 1.00 & & 1.00 & \\
\hline & Yes & $97(20.4 \%)$ & $1.54(0.90-2.62)$ & 0.008 & $0.80(0.39-1.64)$ & 0.539 \\
\hline \multirow{3}{*}{$\begin{array}{l}\text { Have you, anyone in your family or any of your } \\
\text { friends had cancer? }\end{array}$} & No & $84(24.2 \%)$ & 1.00 & & 1.00 & \\
\hline & Yes, self & $42(12.1 \%)$ & $\begin{array}{l}0.34 \\
(0.15-0.75)\end{array}$ & $<0.001$ & $0.70(0.19-2.57)$ & 0.595 \\
\hline & Yes, other & $267(14.8 \%)$ & $\begin{array}{l}0.47 \\
(0.27-0.81)\end{array}$ & $<0.001$ & $0.98(0.45-2.16)$ & 0.967 \\
\hline \multirow{2}{*}{$\begin{array}{l}\text { Did you go for bowel screening the last time you } \\
\text { were invited? }\end{array}$} & Yes & $61(3.0 \%)$ & 1.00 & & 1.00 & \\
\hline & No & $288(80.5 \%)$ & $\begin{array}{l}118.0 \\
(54.88-253.9)\end{array}$ & $<0.001$ & $\begin{array}{l}66.35 \\
(33.45-131.61)\end{array}$ & $<0.001$ \\
\hline Total number barriers, mean (SD) & & $1.01(1.26)$ & $\begin{array}{l}4.47 \\
(2.56-7.81)\end{array}$ & $<0.001$ & $1.59(0.96-2.63)$ & 0.072 \\
\hline \multirow{2}{*}{$\begin{array}{l}\text { I am confident I would be safe from coronavirus } \\
\text { if I needed to attend an appointment at a } \\
\text { hospital }\end{array}$} & $\begin{array}{l}\text { Strongly/somewhat } \\
\text { agree }\end{array}$ & $230(14.0 \%)$ & 1.00 & & 1.00 & \\
\hline & $\begin{array}{l}\text { Strongly/somewhat } \\
\text { disagree }\end{array}$ & $117(19.3 \%)$ & $1.65(0.99-2.76)$ & 0.001 & $0.97(0.38-2.53)$ & 0.958 \\
\hline \multirow{2}{*}{$\begin{array}{l}\text { I am confident I would be safe from coronavirus } \\
\text { if I needed to attend an appointment at my GP } \\
\text { surgery }\end{array}$} & $\begin{array}{l}\text { Strongly/somewhat } \\
\text { agree }\end{array}$ & $259(13.7 \%)$ & 1.00 & & 1.00 & \\
\hline & $\begin{array}{l}\text { Strongly/somewhat } \\
\text { disagree }\end{array}$ & $91(21.7 \%)$ & $\begin{array}{l}2.02 \\
(1.16-3.52)\end{array}$ & $<0.001$ & $0.90(0.35-2.31)$ & 0.824 \\
\hline \multirow[t]{2}{*}{$\begin{array}{l}\text { I am worried about delays to cancer tests and } \\
\text { investigations caused by corona virus }\end{array}$} & $\begin{array}{l}\text { Strongly/somewhat } \\
\text { agree }\end{array}$ & $246(12.9 \%)$ & 1.00 & & 1.00 & \\
\hline & $\begin{array}{l}\text { Strongly/somewhat } \\
\text { disagree }\end{array}$ & $90(22.6 \%)$ & $\begin{array}{l}2.18 \\
(1.26-3.78)\end{array}$ & $<0.001$ & $1.86(0.79-4.39)$ & 0.156 \\
\hline \multirow{2}{*}{$\begin{array}{l}\text { I am less likely to attend a cancer screening } \\
\text { appointment now than I was before the } \\
\text { pandemic }\end{array}$} & $\begin{array}{l}\text { Strongly/somewhat } \\
\text { agree }\end{array}$ & $129(27.7 \%)$ & 1.00 & & 1.00 & \\
\hline & $\begin{array}{l}\text { Strongly/somewhat } \\
\text { disagree }\end{array}$ & $207(11.2 \%)$ & $\begin{array}{l}0.37 \\
(0.22-0.62)\end{array}$ & $<0.001$ & $0.54(0.27-1.07)$ & 0.076 \\
\hline \multirow[t]{2}{*}{$\begin{array}{l}\text { I am worried about delays to cancer screening } \\
\text { caused by coronavirus }\end{array}$} & $\begin{array}{l}\text { Strongly/somewhat } \\
\text { agree }\end{array}$ & $233(12.4 \%)$ & 1.00 & & 1.00 & \\
\hline & $\begin{array}{l}\text { Strongly/somewhat } \\
\text { disagree }\end{array}$ & $99(23.2 \%)$ & $\begin{array}{l}2.59 \\
(1.52-4.41)\end{array}$ & $<0.001$ & $1.51(0.71-3.21)$ & 0.280 \\
\hline \multirow[t]{2}{*}{ Cancer screening saves lives } & $\begin{array}{l}\text { Strongly/somewhat } \\
\text { agree }\end{array}$ & $356(14.7 \%)$ & 1.00 & & 1.00 & \\
\hline & $\begin{array}{l}\text { Strongly/somewhat } \\
\text { disagree }\end{array}$ & $11(29.0 \%)$ & $\begin{array}{l}3.42 \\
(0.85-13.73)\end{array}$ & 0.004 & $1.75(0.55-5.58)$ & 0.346 \\
\hline
\end{tabular}

\footnotetext{
$\mathrm{p}<0.001$.

$p<0.01$.
} 
more frequently reported by participants who had not been screened when last invited (see Supplementary Material 3).

\subsubsection{Correlates of future cervical screening intention}

In unadjusted analyses (see Table 2), low cervical screening intention was statistically significantly associated with being from an ethnic minority background (compared with being white), being single (compared with being married/in a relationship), living in England (compared with Wales), not taking part in cervical screening when last invited and endorsing more cervical screening barriers. Concerns about COVID-19 when visiting a hospital or GP surgery were associated with lower intentions to attend screening, as was being less likely to attend a cancer screening appointment now (i.e., during the pandemic) than before. Not being worried about COVID-related delays to cancer tests and screening and not agreeing that cancer screening saves lives were associated with lower intention to attend screening. In the fully adjusted model, being single, living in England (compared with Wales), previous cervical screening non-attendance, reporting more barriers to screening and being less likely to attend a cancer screening appointment now than before the pandemic remained statistically significant predictors of low future intention.

\subsubsection{Correlates of future CRC screening intention}

In unadjusted analyses (see Table 3), low CRC screening intention was statistically significantly associated with being single, living in England, not having previous experience of cancer (compared with own experience or that of a friend/family), not having completed a CRC screening kit when last invited and endorsing more CRC screening barriers. Being concerned about COVID-19 when visiting the GP surgery, not being worried about COVID-related delays to cancer tests and screening and being less likely to attend a cancer screening appointment now were associated with lower intention. In the fully adjusted model, only past screening non-participation remained significantly associated with low intention to take part in future.

\subsection{Qualitative results}

\subsubsection{Sample characteristics}

Thirty participants were interviewed. Seventeen were male and 19 had a higher education qualification or degree. Most lived in Wales ( $\mathrm{n}=$ $25)$ and were from a White ethnic background $(n=23)$. Mean age was 55 years (range: $26-76)$. Most were eligible for either cervical $(\mathrm{N}=11)$ and/or CRC screening $(N=11)$, with 9 not currently eligible for either. Exemplar quotes and definitions of key themes are shown in Table 4.

\subsubsection{Views on changes to screening prior to and during the pandemic}

Most eligible participants reported engaging in cancer screening prior to the pandemic. Participants were supportive of the national screening programmes and considered them important.

There was varying awareness that screening had effectively been paused. Those who were aware often understood the decision, though many expressed hope that lessons could be learned to avoid pausing screening again in the future. Participants' understanding of the pause was frequently linked to their trust in the NHS; they trusted that doctors had made the right decision. Others questioned the need for changes as procedures and tests are often performed away from hospitals in the GP practice. These participants also expressed concerns that although COVID-19 was a priority, other conditions should not be neglected.

Participants reiterated the message that the public should be treated as adults, valuing open and honest communication about decisions on screening. They felt that this had not happened.

\subsubsection{Fears about screening during the pandemic}

Screening fears were related to the need to attend at a healthcare setting. Participants were not fearful of completing the CRC screening test if they had received one. Though participants mentioned some of the
Table 4

Exemplary participant quotes and theme definitions by major theme, UK, August-September 2020.

\begin{tabular}{ll}
\hline Major theme & Theme definition \\
& \\
\hline $\begin{array}{l}\text { Engagement in } \\
\text { screening, and } \\
\text { screening during the } \\
\text { pandemic }\end{array}$ & $\begin{array}{l}\text { Any discussion or } \\
\text { reference as to whether } \\
\text { and why participants are } \\
\text { engaged in screening } \\
\text { programmes or not. } \\
\text { Whether the participant } \\
\text { was aware of the changes } \\
\text { to screening due to the } \\
\text { pandemic. Whether any } \\
\text { of their views/opinions of } \\
\text { screening had changed } \\
\text { during the pandemic or } \\
\text { associated lockdowns. }\end{array}$ \\
\hline
\end{tabular}

Exemplary participant quotes (participant ID, gender, age (years), nation of residency) [Quotes provided in intelligent verbatim; $\mathrm{P}=$ Participant, I = Interviewer]

P: I've had the colon bowel cancer thing screening which was one of the most uncomfortable experiences but I did have it cos I did see the benefit of it. (64052923, F, 57, England)

I: So you'd said that it would concern you if, for instance the screening programmes didn't resume. So does that mean that you value the screening programmes? Do you think they're very important? P: Yes, I do. Because obviously with, with any ailment or disease the sooner it's found I guess the, the better survival is and the rest, but not everything is... I trust that they wouldn't have these things for no good reason, you know? (64026131, M, 62, Wales)

P: I think the tests and the scans are useful, and I think it's disappointing that they can't continue, but I can fully understand that they're more like difficult to continue. (63982840, M, 73, Wales)

P: I mean, I don't think it should be a flexible option, because I think it can save people's lives, and so I think it's something that they should prioritise and go forward with. Um, and that's why they you know, advertise quite heavily for us to get the screening. (64054578, F, 26, England)

P: ... so, um, I had, um, appointments booked, it was sort of March/April time, for my smear, for my mammogram. And of course, then, as we went into lockdown, all these letters were coming, to cancel the appointments. And that's a bit disheartening, isn't it, when you feel as if you want to act on something ... (63984570, F, 53, Wales)

I: So you had been invited for screening, was that (continued on next page) 
Table 4 (continued)

\begin{tabular}{|c|c|c|}
\hline Major theme & Theme definition & $\begin{array}{l}\text { Exemplary participant } \\
\text { quotes (participant ID, } \\
\text { gender, age (years), nation } \\
\text { of residency) [Quotes } \\
\text { provided in intelligent } \\
\text { verbatim; } \mathrm{P}=\text { Participant, } \\
\mathrm{I}=\text { Interviewer] }\end{array}$ \\
\hline & & $\begin{array}{l}\text { before the lockdown or just } \\
\text { round about the same } \\
\text { time? P: Yeah, it was just } \\
\text { before. I think by the time I } \\
\text {... I think it was about } \\
\text { maybe a week or a few } \\
\text { weeks before... I mean my } \\
\text { norm. I hate them, so my } \\
\text { normal thing is to delay it a } \\
\text { little bit to be honest... so, } \\
\text { you know the fact that I } \\
\text { kind of, oh that's } \\
\text { something I'll look at a } \\
\text { little bit later.. is probably } \\
\text { quite a normal thing and } \\
\text { then that little bit later } \\
\text { became a lot later because I } \\
\text { knew that I wasn't going to } \\
\text { be possible. Well I didn't } \\
\text { know, but I just assumed it } \\
\text { wasn't going to be possible } \\
\text { (64078317, F, } 46 \text {, England) }\end{array}$ \\
\hline & & $\begin{array}{l}\text { P: I'm not sure to be honest } \\
\text { because like I said it is, it is } \\
\text { important that people are } \\
\text { screened and by the point } \\
\text { we are now, where we're } \\
\text { sort of six or seven months } \\
\text { into this, that's a lot of } \\
\text { people who have been } \\
\text { missed... so going forward } \\
\text { I think something does } \\
\text { need to be done to address } \\
\text { that, that maybe } \\
\text { screenings need to be done } \\
\text { at different locations or } \\
\text { they need to be more } \\
\text { caretaking, I don't know } \\
\text { what caretaking is at the } \\
\text { moment, you know, to stop } \\
\text { the spread of things... but } \\
\text { obviously you can't really } \\
\text { be, you can't stop } \\
\text { screenings because they're } \\
\text { there for a reason in the } \\
\text { first place, but we need to } \\
\text { find a way to be able to do } \\
\text { them going forward while } \\
\text { the pandemic is still } \\
\text { around because I can't see } \\
\text { this going away any time } \\
\text { soon ( } 64027453, M, 38 \text {, } \\
\text { Wales) }\end{array}$ \\
\hline $\begin{array}{l}\text { Fears about screening } \\
\text { during the pandemic }\end{array}$ & $\begin{array}{l}\text { Any discussion or } \\
\text { reference to the } \\
\text { participants thoughts, } \\
\text { feelings or views of } \\
\text { attending or completing } \\
\text { (actual or hypothetical) } \\
\text { screening during the } \\
\text { pandemic and associated } \\
\text { lockdowns }\end{array}$ & $\begin{array}{l}\text { P: I've had stuff about } \\
\text { screening as well although } \\
\text { they're not offering } \\
\text { screening. Because I was } \\
\text { due to have a smear test in } \\
\text { February but I cancelled it } \\
\text { because I didn't feel } \\
\text { comfortable going, } \\
\text { knowing about the } \\
\text { pandemic. It was right at } \\
\text { the end of February and I } \\
\text { thought, actually I'm not } \\
\text { sure about that.... I felt } \\
\text { like the, the risk to my } \\
\text { health at that point } \\
\text { outweighed the benefit of }\end{array}$ \\
\hline
\end{tabular}

Table 4 (continued)

\begin{tabular}{ll}
\hline Theme definition & Exemplary participant \\
quotes (participant ID, \\
gender, age (years), nation \\
of residency) [Quotes \\
provided in intelligent \\
verbatim; P = Participant, \\
I = Interviewer] \\
having the screening \\
(63959480, F, 28, England) \\
\\
I: ... would you have \\
thought it was safe to go \\
for screening? P: If it was \\
possible, as I've spoken to \\
someone who was working \\
there, and asked what \\
measures they were taking, \\
what measures it was \\
advised I take. And then I'd \\
probably show up and \\
have a look at how bad it \\
is... But the way everyone's \\
been basically carrying on. \\
Like keeping their distance \\
and being gowned and \\
masked and, you know, it \\
would, you'd be waiting in \\
your car, you'd be \\
attending, you'd be \\
leaving. It would be... you \\
know, everything's a risk, \\
isn't it? But going to the \\
screening is the problem. \\
Not having the screening \\
(63977972, F, 32 Wales) \\
\end{tabular}

P: Yeah, I mean probably not if they hadn't cancelled the appointment. I mean I would have had the normal concerns which I've said about, you know, just getting out and about and the risks and stuff like that. But that just seems to be like part of the ... that's normal now. I get on a bus, tube every week and I have concerns that I'll pick something up but not enough to stop me... Erm, I think that I'd have relied on the provider, that I'd have been reassured that if my appointment wasn't cancelled and therefore they deemed it safe then it was safe (64078317, F, 46, England)

I:... would you have had concerns about attending if you were due to attend? P: I would have certainly, because there was so much unknown about the virus and how easily it was transmitting and that, that close person contact that.. You know I wouldn't have felt comfortable at that stage. I: Okay, would you have had concerns about getting an infection perhaps? I: Yes contracting COVID at the centre and

(continued on next page) 
Table 4 (continued)

\begin{tabular}{ll}
\hline Major theme & Theme definition \\
& \\
\hline Screening recovery & \\
& Any views, suggestions or \\
& ideas provided by \\
participants to support \\
and/or encourage \\
screening attendance or \\
screening completion. \\
Any views, suggestions or \\
ideas of practical re- \\
opening and running of \\
screening services after \\
pandemic, or during \\
continued lockdown \\
measures.
\end{tabular}

Exemplary participant quotes (participant ID, gender, age (years), nation of residency) [Quotes provided in intelligent verbatim; $\mathrm{P}=$ Participant, $\mathrm{I}=$ Interviewer]

then taking it back, yeah (64948240, F, 46, Wales)

$\mathrm{P}$ : I think being prompted by the GP... and also knowing about the measures that they're putting in place to try and keep people safe about social distancing during that and the other things we've mentioned... Yeah, so it's about making people aware of the importance of getting it done, and how safe it will be (63959480, $F, 28$, England)

I:...so you mentioned that you were due to have cervical screening during the lockdown, but you were told that it couldn't take place because of COVID. P: Yeah, which I was a bit put off by, because it said I was due to have one done now. I: Yeah, so have you been contacted again now, to have that done? P: No and I don't know what to do about it. I don't know whether I've got to phone them myself, but then it's the rigmarole of going by wheelchair again, getting up to the doctors, which is a bit of a pain like (64018114, F, 44, Wales)

P: Erm, I think again it does ... it's the information and the messaging isn't it?... I think if I'd just had a letter, come in and do this, I think I probably would have been concerned. But if it was with more detail, with and this is how we'll be doing it and you will need to do $\mathrm{a}, \mathrm{b}$, and $\mathrm{c}$ and we will do a, b and c, erm. But also, they need, I think there needed to be a sort of caveat that if you're really not happy about coming we're not going to ... you're not going to fall off the list, we'll put you back on and keep asking you (63984720, F, 46, Wales)

P: Well that's going to be difficult... I would say it should go in date order...if nobody's been screened then it should revert to date order, but if people were halfway through their screening process and
Table 4 (continued)

\begin{tabular}{ll}
\hline Major theme definition & $\begin{array}{l}\text { Exemplary participant } \\
\text { quotes (participant ID, } \\
\text { gender, age (years), nation } \\
\text { of residency) [Quotes } \\
\text { provided in intelligent } \\
\text { verbatim; } \mathrm{P}=\text { Participant, } \\
\mathrm{I}=\text { Interviewer] }\end{array}$ \\
\hline they've actually done tests \\
then those with the worst \\
health condition should be \\
invited in first and those \\
people that, when the tests \\
were done, their health \\
was not as bad as others, \\
maybe should be invited in \\
afterwards (64,052,923, $F$, \\
57, England)
\end{tabular}

P: It's the reassurance really of what additional measures are in place. You know in addition to the existing measures.. Because you're told about ventilating rooms and like you, you certainly wouldn't want cervical screening undertaken with the door open [laughs] or, you know, whatever... it is now that very close, intimate contact. How on earth, can you manage not to, not to present a risk in that situation (64948240, F, 46, Wales)

P: I think that a slightly more adult approach by the NHS to people's expectations and, and instead of just getting a letter thing, "You're now due for screening"... you know, it wouldn't be too difficult or it's possible to say, "we had to put screening on hold." this doesn't reduce the importance or the necessity of the screening, it was just a decision to ensure that we had maximum protection for people. And avoid any cross, cross-infection during COVID... But now that we've taken these steps A, B and C to ensure your protection during the next round of screening, we very much need you to come along because it's still as important as it once was... I think an explanation along those lines would inform people and enable people and I think the more, er, information, the more informed a person is, the better on, better able they are to make a choice (63968088, M, 61, Wales)

P: Yeah, it's a tricky one, isn't it, because obviously

(continued on next page) 
Table 4 (continued)

\begin{tabular}{ll}
\hline Major theme & Theme definition \\
& quotes (participant ID, \\
& gender, age (years), nation \\
& of residency) [Quotes \\
& provided in intelligent \\
& verbatim; P = Participant, \\
& $\mathrm{I}=$ Interviewer] \\
\hline
\end{tabular}

the Government are going to try, or the health people, you know, a particular section of health, are going to try and say how it was and is. And sceptical people will say, "Well, it wasn't that important, was it, cos you cancelled it. So I think, um, I think they've given themselves a bit of a problem there. I think what you, we have to say is that it was impossible to continue it, but now it's started again. And it's very important that people take up the opportunity to, for their own health (64021806, F, 64, Wales)

well-known barriers to completing the stool sample such as it being unpleasant, they expressed no specific COVID-related concerns. However, worries were heightened over cervical screening attendance (though not the procedure itself) due to its completion in a healthcare setting. This was the same for those who had been invited to screening during the pandemic and those who were asked hypothetically.

There was general fear or nervousness about attending healthcare settings. Fears were associated with the 'unknown' (understanding and negotiating new systems), catching COVID-19 or passing it on to others, encountering other members of the public not adhering to social distancing and using public transport. Participants described having to weigh up the risks of attendance versus not attending in making their decision.

Some of those affected voiced relief that their cervical screening appointment had been delayed or cancelled on their behalf, relieving them of the responsibility of deciding. Others expressed that to help relieve pressures for the NHS in the future (due to potential delayed diagnosis) the least they could do is to attend cervical screening when invited.

\subsubsection{Screening recovery}

Participants who had had screening delayed expressed confusion about whether they would be contacted to rearrange screening appointments, or whether they themselves should proactively contact services. Enthusiasm for rearranging appointments was hindered by worry that they would be bothering already stretched services. To counteract fears of COVID, participants suggested that GP practices provide information on their new infection control practices, so patients knew what to expect. When considering the backlog of those who missed screening, consensus was that this should be managed based on clinical need and risk.

\section{Discussion}

The COVID-19 pandemic has led to widespread disruption of cancer screening. The current study is, to our knowledge, the first to show high future intentions to take part in cervical and CRC screening in the UK during the pandemic. Past non-attendance and (for cervical screening) endorsement of barriers were the strongest correlates of low future intentions to take part. For cervical screening, intention to participate was lower among women who had not attended their last screen for reasons unrelated to COVID-19. Despite overall intentions being high, a substantial minority of participants stated that they were less likely to attend a screening appointment than before the pandemic.

Our finding that $74 \%$ of women definitely intended to go for cervical screening (rising to $86 \%$ if the 'yes, probably' response was included) is comparable with Marlow et al., (2017) who found 88\% of women in England intended to be screened using a yes/no outcome. For CRC screening, $84 \%$ of our sample definitely intended to take part, which is considerably higher than the $64 \%$ reported in Dodd et al. (2019) survey using similar methods. Despite these reassuringly high levels of intention, the finding that $30 \%$ of the cervical-eligible and $19 \%$ of the CRCeligible samples said they were less likely to attend a screening appointment now than prior to the pandemic is concerning. This reticence is more relevant to cervical screening than home-based CRC screening and may go some way to explaining the unexpectedly lower intention for cervical than CRC screening (which is at odds with data on actual screening uptake in the two programmes; uptake rates in England were $72 \%$ for cervical screening in 2019/2020 and 64\% for CRC screening in 2020 (Public Health England, NHS Digital, 2020; Public Health England, 2021).

Future cervical screening intentions were higher in women who reported not attending their last cervical screening for COVID-related reasons, compared with those whose reasons were not related to the pandemic. However, intentions among women who chose not to attend were lower than those who were unable to attend due to COVID-19. In addition, lower intention was associated with being less likely to attend a cancer screening appointment than before the pandemic. Directly experiencing screening disruption during the pandemic may therefore have had an impact on willingness to attend in the future. It is encouraging that screening in the UK has been much less disrupted during subsequent lockdowns but efforts may be needed to ensure those who have missed cervical screens are provided with additional support. Interview participants expressed uncertainty over the process for rebooking missed cervical screening and wished to resume screening when available.

In multivariable analyses, screening intentions were no lower in participants who were worried about visiting healthcare settings during the pandemic than those who were not worried. However, qualitative data suggested that some people did have COVID-related concerns including travelling to and attending healthcare settings, and fear of COVID-19 infection (Rees et al., 2020). As previous screening uptake was high in this sample, few barriers to screening were reported in the survey. Barriers that were endorsed mirrored those reported in prepandemic research including disgust, embarrassment, prior screening experiences and fear of what might be found (Waller et al., 2009; Chapple et al., 2008; Bennett et al., 2018; von Wagner et al., 2009). High levels of worry about the delays to cancer screening caused by COVID-19 were reported in the survey (though they were not associated with intentions). Recent research has shown the potentially damaging effects of the screening programme pause on cancer detection and survival (Cancer Intelligence Team, 2021; Morris et al., 2021; Sud et al., 2020) and participants' concerns reflected this, perhaps due to media coverage of the impact of the pandemic on cancer services. Of note, data collection for this study (both survey and interview) took place after the first lockdown, although in the latter half of 2020 local lockdowns may have been in place for some participants. Although healthcare visits were permitted throughout lockdowns, attitudes and priorities may have been affected by local restrictions and the 'stay at home' message could have been interpreted as meaning that screening should wait.

\subsection{Study limitations and strengths}

We recruited a large sample from across the UK and weighted data to be representative of the UK for age, gender, ethnicity and region. The data are cross-sectional, and caution must be exercised when 
interpreting the results. In addition, participants' self-reported intentions might not translate into behaviour (Sheeran and Webb, 2016), although we used 'yes, definitely' to indicate positive intention which has demonstrated a strong association with screening behaviour (Power et al., 2008). Intention to complete CRC screening was higher than population-level uptake, and may also reflect the high number of HealthWise Wales participants in the CRC cohort, who may be more health-motivated than the general population. (Chambers et al., 2016) Sample selection bias may also explain high overall screening intentions, with limited power to detect the effects of factors expected to be associated with screening intentions such as education and ethnicity. Interview participants were sampled based on symptom experience (the primary study outcome), hence some were not eligible for screening and their responses were therefore hypothetical.

\subsection{Implications}

The finding that past screening non-attendance was associated with lower future screening intention is not new, but it underlines the continued importance of interventions to reduce COVID and non-COVID screening barriers among non-participants, including women who have not attended cervical screening for COVID-related reasons. During August and September 2020 a significant minority of the population remained wary of visiting healthcare settings due to coronavirus, therefore clear public health messaging is needed to provide reassurance about the safety of attending resumed cancer screening services, prioritising known risk-groups (by disease, socioeconomic group and age) (Castanon et al., 2021b). It is also important to ensure there is sufficient screening and diagnostic workforce capacity, so that follow-up tests can be done in a timely manner. In addition, new adapted technologies for cervical screening have the potential to overcome a range of wellestablished barriers as well as reluctance to attend healthcare settings due to coronavirus risk. The pandemic provides an impetus for expedited implementation of HPV self-sampling to address cervical screening backlogs (Lim, 2021), potentially also increasing screening uptake.

\section{Conclusions}

Despite concerns about attending healthcare settings during the COVID-19 pandemic, intentions to take part in cancer screening appeared to remain high during the pandemic. Efforts to restore screening participation to at least pre-pandemic levels will require clear communication with the public to address safety concerns, as well as strategies to increase screening and diagnostic workforce capacity. Ongoing evaluation is needed to assess whether high intentions are reflected in screening uptake.

\section{Additional information}

Registration: ISRCTN17782018.

\section{Ethics approval}

Ethical approval was granted by the School of Medicine Research Ethics Committee, Cardiff University (ref 20.68).

\section{Data availability}

De-identified participant data will be made available to the scientific community with as few restrictions as feasible, whilst retaining exclusive use until the publication of major outputs. Data will be available via the corresponding author.

\section{Funding information/Role of the funding source}

Economic and Social Research Council as part of UK Research and
Innovation's Rapid Response to COVID-19 (ES/V00591X/1). The funders had no role in the design, conduct or analyses of this study.

\section{Declaration of Competing Interest}

The authors declare no conflict of interest.

\section{Acknowledgments}

This study was facilitated by HealthWise Wales, the Health and Care Research Wales initiative, which is led by Cardiff University in collaboration with SAIL, Swansea University. HQS is funded by PRIME Centre Wales, which is funded by Welsh Government through Health and Care Research Wales. DECIPHer and The Centre for Trials Research receives funding from Health and Care Research Wales, and Health and Care Research Wales and Cancer Research UK respectively. SS is funded by a Health and Care Research Wales fellowship (514366). JW is funded by a Cancer Research UK Career Development Fellowship (C7492/A17219). We are grateful to Cancer Research UK's Cancer Insights Patient Panel, Clinical Advisory Panel and GP Panel for their helpful feedback. Cancer Research UK staff members and other researchers contributed to the initiation and development of the CAM, both historically and for this project. COVID-CAM data was provided by Cancer Research UK who collected the data via Dynata's online survey panels. External Scientific Advisory Group members who advised the statistical analysis plan include: Professor Jamie Brown (University College London), Professor Yoryos Lyratzopoulos (University College London), Dr. Katie Robb (University of Glasgow), Dr. Christian von Wagner (University College London) and Professor Fiona Walter (University of Cambridge).

\section{Appendix A. Supplementary data}

Supplementary data to this article can be found online at https://doi. org/10.1016/j.ypmed.2021.106826.

\section{References}

Bennett, K.F., Waller, J., Chorley, A.J., Ferrer, R.A., Haddrell, J.B., Marlow, L.A.V., 2018. Barriers to cervical screening and interest in self-sampling among women who actively decline screening. J. Med. Screen. 25 (4), 211-217.

Braun, V., Clarke, V., 2006. Using thematic analysis in psychology. Qual. Res. Psychol. 3 (2), 77-101.

CABS, 2021. Covid Health and Help-seeking Study (CABS) [Internet] [cited 2021 Mar 9]. Available from: Retrieved from osf.io/zxyp3.

CAM, 2021. COVID Cancer Awareness Measure (COVID-CAM) wave 12020 [Internet] [cited 2021 Mar 25]. Available from: https://osf.io/j67dt/.

Cancer Intelligence Team, 2021. Evidence of the Impact of COVID-19 across the Cancer Pathway : Key Stats.

Castanon, A., Rebolj, M., Pesola, F., Sasieni, P., 2021a. Recovery strategies following COVID-19 disruption to cervical cancer screening and their impact on excess diagnoses. Br. J. Cancer. [internet]. https://doi.org/10.1038/s41416-021-01275-3 (January):1-5. Available from:

Castanon, A., Rebolj, M., Burger, E.A., De, Kok I.M.C.M., Smith, M.A., SJB, Hanley, et al., 2021b. Viewpoint cervical screening during the COVID-19 pandemic : optimising recovery strategies. Lancet Public Heal. [Internet]. 2667 (21), 1-6. Available from: https://doi.org/10.1016/S2468-2667(21)00078-5.

Chambers, J.A., Callander, A.S., Grangeret, R., O'Carroll, R.E., 2016. Attitudes towards the faecal occult blood test (FOBT) versus the faecal immunochemical test (FIT) for colorectal cancer screening: perceived ease of completion and disgust. BMC Cancer [Internet]. 16 (1), 1-7. Available from: https://doi.org/10.1186/s12885-0 16-2133-4.

Chapple, A., Ziebland, S., Hewitson, P., McPherson, A., 2008. What affects the uptake of screening for bowel cancer using a faecal occult blood test (FOBt): a qualitative study. Soc. Sci. Med. 66 (12), 2425-2435.

de Jonge, L., Worthington, J., van Wifferen, F., Iragorri, N., Peterse, E.F.P., Lew, J.-B., et al., 2021. Impact of the COVID-19 pandemic on faecal immunochemical test-based colorectal cancer screening programmes in Australia, Canada, and the Netherlands: a comparative modelling study. Lancet Gastroenterol. Hepatol. 6 (April).

Dodd, R.H., Kobayashi, L.C., von Wagner, C., 2019. Perceived life expectancy and colorectal cancer screening intentions and behaviour: a population-based UK study. Prev. Med. Rep. [Internet]. 16 (September), 101002. Available from: https://doi.or g/10.1016/j.pmedr.2019.101002.

Douglas, E., Waller, J., Duffy, S.W., Wardle, J., 2016. Socioeconomic inequalities in breast and cervical screening coverage in England: are we closing the gap? J. Med. 
Screen [Internet]. 23 (2), 98-103. Available from: www.kingsfund.org.uk/blog /2012/07/ccgs-and-pcts-not-so-different.

Hudson, B., Osborne, K., 2021. Cancer Research UK's Cancer Awareness Measure 2019 [Internet]. Available from: osf.io/zd2sv.

Ide-Walters, C., Moffat, J., 2021. COVID - Survey - Confidence to Attend Appointments and Tests at Primary and Secondary Care Settings [Internet]. Available from: htt ps://osf.io/km4ry/.

Jones, D., Neal, R.D., Duffy, S.R.G., Scott, S.E., Whitaker, K.L., Brain, K., 2020. Impact of the COVID-19 pandemic on the symptomatic diagnosis of cancer: the view from primary care [Internet]. In: The Lancet Oncology, Vol. 21. Lancet Publishing Group, pp. 748-750 [cited $2021 \mathrm{Feb} 10$ ]. Available from: www.thelancet.com/oncology.

Lim, A.W.W., 2021. Will COVID-19 be the tipping point for primary HPV self-sampling? Cancer Epidemiol. Biomark. Prev. 30 (2), 245-247.

Marlow, L.A.V., Chorley, A.J., Haddrell, J., Ferrer, R., Waller, J., 2017. Understanding the heterogeneity of cervical cancer screening non-participants: data from a national sample of British women. Eur. J. Cancer [Internet]. 80, 30-38. Available from: https://www.sciencedirect.com/science/article/pii/S095980491730919X.

Morris, E.J.A., Goldacre, R., Spata, E., Mafham, M., Finan, P.J., Shelton, J., et al., 2021 Impact of the COVID-19 pandemic on the detection and management of colorectal cancer in England: a population-based study. Lancet Gastroenterol. Hepatol. [internet]. 6 (3), 199-208. Available from: https://doi.org/10.1016/S2468-1253 (21)00005-4.

Palmer, C.K., Thomas, M.C., Von Wagner, C., Raine, R., 2014. Reasons for non-uptake and subsequent participation in the NHS bowel cancer screening programme: a qualitative study. Br. J. Cancer 110 (7), 1705-1711.

Power, E., Van Jaarsveld, C.H.M., McCaffery, K., Miles, A., Atkin, W., Wardle, J., 2008. Understanding intentions and action in colorectal cancer screening. Ann. Behav. Med. 35 (3), 285-294.

Public Health England, 2021. Public Health Profiles [Internet] [cited 2021 Apr 1]. Available from: https://fingertips.phe.org.uk/search/cancerscreening.

Public Health England, NHS Digital, 2020. Cervical screening programme, England 2019-20 [Internet] [cited 2021 Apr 1]. Available from: https://digital.nhs. uk/data-and-information/publications/statistical/cervical-screening-annual/en gland-2019-20/author-copyright-and-licensing.

QSR International Pty Ltd, 2018. NVivo Qualitative Data Analysis Software.

Quinn-Scoggins, H., Cannings-John, R., Moriarty, Y., Whitelock, V., Whitaker, K.L.,

Grozeva, D., et al., 2021. The Impact of COVID-19 on Cancer Symptom Experience and Help-Seeking Behaviour in the United Kingdom: A Cross-Sectional Population Survey [Internet]. SSRN. [cited 2021 Mar 16]. Available from: https://papers.ssrn. $\mathrm{com} /$ sol3/papers.cfm?abstract_id=3793564.

Rees, C.J., Rutter, M.D., Sharp, L., Hayee, B., East, J.E., Bhandari, P., et al., 2020. COVID19 as a barrier to attending for gastrointestinal endoscopy: weighing up the risks. Lancet Gastroenterol. Hepatol. [Internet]. 5 (11), 960-962. Available from: https://www.thelancet.com/journals/langas/article/PIIS2468-1253(20)30268-5 /fulltext.

Sheeran, P., Webb, T.L., 2016. The intention-behavior gap. Soc. Personal Psychol. Comp. [Internet]. 10 (9), 503-518. Available from: http://eprints.whiterose.ac. uk/107519/.

Smith, S.G., McGregor, L.M., Raine, R., Wardle, J., von Wagner, C., Robb, K.A., 2016 Inequalities in cancer screening participation: examining differences in perceived benefits and barriers. Psychooncology. 1168-1174 (December 2015).

StataCorp, 2019. Stata Statistical Software: Release 16. StataCorp LLC, College Station, TX.

Sud, A., Torr, B., Jones, M.E., Broggio, J., Scott, S., Loveday, C., et al., 2020. Effect of delays in the 2-week-wait cancer referral pathway during the COVID-19 pandemic on cancer survival in the UK: a modelling study. Lancet Oncol. [Internet]. 21 (8), 1035-1044. Available from: https://doi.org/10.1016/S1470-2045(20)30392-2.

von Wagner, C., Good, A., Wright, D., Rachet, B., Obichere, A., Bloom, S., et al., 2009 Dec 3. Inequalities in colorectal cancer screening participation in the first round of the national screening programme in England. Br. J. Cancer [Internet]. 101 (S2), S60-S63 [cited 2017 Dec 18]. Available from: http://www.nature.com/doifi nder/10.1038/sj.bjc.6605392.

Vrinten, C., Stoffel, S., Dodd, R.H., Waller, J., Lyratzopoulos, Y., von Wagner, C., 2019. Cancer worry frequency vs. intensity and self-reported colorectal cancer screening uptake: a population-based study. J. Med. Screen. 26 (4), 169-178.

Waller, J., Bartoszek, M., Marlow, L., Wardle, J., 2009 Jan 1. Barriers to cervical cancer screening attendance in England: a population-based survey. J. Med. Screen [Internet]. 16 (4), 199-204 [cited 2016 Jul 8]. Available from: http://msc.sagepub. com/content/16/4/199.full.

World Health Organization, 2017. Guide to Cancer - Early Diagnosis [Internet]. World Health Organization, p. 48 [cited 2021 Mar 16]. Available from: https://apps.who. int/iris/bitstream/handle/10665/254500/9789241511940-eng.pdf;jsessionid=26 46A3E30075DB0FCA4A703A481A5494? sequence $=1$. 